\title{
PROGRESS ON USING NEA CATHODES IN AN RF GUN*
}

\author{
R.P. Fliller III", T. Anderson, H. Edwards, Fermilab, Batavia, IL 60510, U.S.A \\ H. Bluem, T. Schultheiss, AES, Medford, NY 11763, U.S.A \\ C. Sinclair, Cornell University, Ithaca, NY 14853, U.S.A \\ M. Huening, DESY, Hamburg
}

\begin{abstract}
RF guns have proven useful in multiple accelerator applications, and are an attractive electron source for the ILC. Using a NEA GaAs photocathode in such a gun allows for the production of polarized electron beams. However the lifetime of a NEA cathode in this environment is reduced by ion and electron bombardment and residual gas oxidation. We report progress made with studies to produce a RF gun using a NEA GaAs photocathode to produce polarized electron beams. We discuss simulations of ion back bombardment and attempts to reduce the residual gas pressure in the gun are discussed. Future directions are also discussed.
\end{abstract}

\section{INTRODUCTION}

The ILC requires an electron source with the ability to produce high brightness, polarized beams. RF electron guns have a proven track record of producing high brightness beams. Experiments conducted at Fermilab have shown the ability of producing beams with a high emittance ratio in an RF gun that can simplify damping ring design [1]. However, the ability to produce polarized beams with an RF gun has been problematic.

Polarized electrons can be produced using NEA GaAs photocathodes. A monolayer of cesium oxide in the surface of the cathode lowers the work function at the surface allowing polarized electrons to tunnel out of the cathode. This layer of cesium oxide is subject to damage from residual gas oxidation and ion bombardment [2].

In this paper we discuss simulations of ion bombardment using the particle-in-cell code MAGIC [3]. We also discuss attempts to reduce the residual gas pressure in an RF gun by cooling it with liquid nitrogen. We compare the dark current in a cryogenically cooled gun with a room temperature gun as well.

\section{ION BOMBARDMENT SIMULATIONS}

There are two sources of electrons within the gun that can lead to ion production. They are field emitted electrons (dark current), and the photo-emitted electron beam.

The electron beam was modeled with a single short electron pulse produced at the appropriate RF phase. The dark current electrons were produced using the built-in

\footnotetext{
* This work was supported by Universities Research Association Inc. under contract DE-AC02-76CH00300 with the U.S. DOE and by NICADD.

AES personnel were supported under DOE SBIR contract \#DEFG02-04ER838.

\# Email: fliller@fnal.gov
}

field emission model ensuring appropriate timing of dark current electrons. The ions were produced via a built in ionization algorithm that statistically generates ions based upon a specified background gas species and pressure using an impact ionization model that takes into account the energy dependent ionization cross-section.

A single $1 \mathrm{nC}$ electron pulse was used to model the primary electron beam. However, at UHV pressures, a single $1 \mathrm{nC}$ electron bunch propagating through the gun will not produce a significant quantity of ions. Thus, to achieve a statistically significant result, the residual gas pressure was increased by several orders of magnitude. The results are then scaled to $1 \times 10^{-10}$ torr.

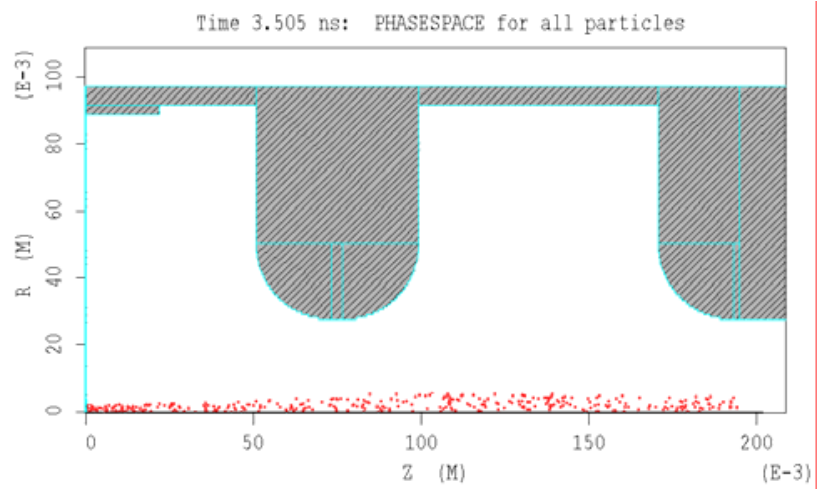

Figure 1: Distribution of ions just after electron beam pulse has exited simulation area.

Figure 1 shows the "initial" ion distribution just after the electron pulse has exited the simulation area. The ions are red in color. The background gas, consisting only of $\mathrm{H}_{2}$, was constrained to the interior of the RF cavities, to help conserve simulation time, thus the abrupt end of the ions at the exit of the second cell.

Figure 2 shows a sampling of the ion trajectories after the simulation. One fifth of the trajectories, randomly chosen by MAGIC, are shown in the figure. One can clearly see the dividing points within each cell where the ions drift in opposite directions, causing the ions to accumulate near the irises. Ions impacting the cathode plane are generated within roughly $10-12 \mathrm{~mm}$ of the cathode. No ions from the second cell appear to drift back to the cathode.

The simulations also provided the total ion charge and average ion kinetic energy impacting the cathode as a function of time. Scaling the numbers obtained in the simulation to a vacuum level of $1 \times 10^{-10}$ torr, one obtains approximately 0.03 ions impacting the cathode per $1 \mathrm{nC}$ electron pulse or $3 \times 10^{7}$ ions per Coulomb. In contrast, a DC gun produces the same number of ions while 
operating with a vacuum level of $1 \times 10^{-11}$ torr [4]. These numbers indicate an order of magnitude advantage for the RF gun in terms of numbers of ions impacting the cathode as a function of vacuum pressure.

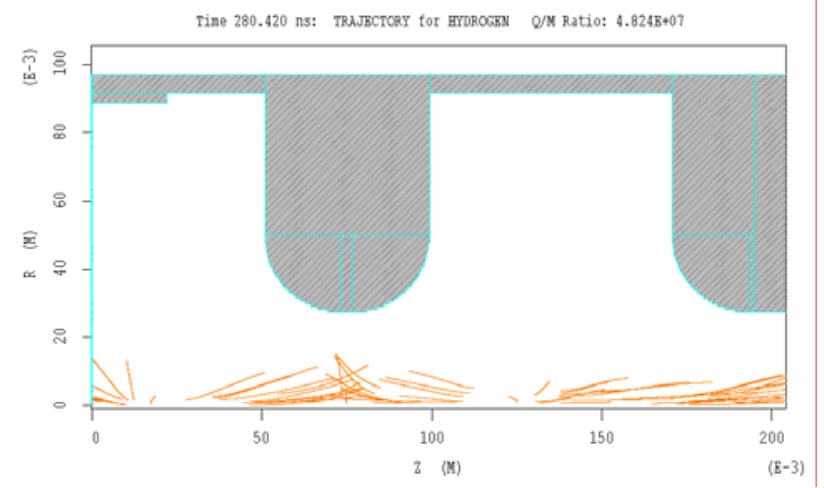

Figure 2: Trajectories through the simulation of a random sample of the ions.

Ion impact energy is also important with higher energy ions causing more damage than low energy ions. Impact energies are $100 \mathrm{keV}$ in DC guns, compared to $2 \mathrm{keV}$ energies seen in simulations of an RF gun. This combined with the smaller number of impacting ions at a given vacuum pressure, there seems to be a potentially significant advantage for RF guns over DC guns in regards to ion bombardment.

Dark currents, electron back-bombardment, and their associated ion generation were also studied using MAGIC. Dark current was allowed to be generated over $10 \mathrm{RF}$ periods using a field emission model with a work function of $1.42 \mathrm{eV}$ with a field enhancement factor of 50 .

Simulations indicated that, for a flat cathode backplane, only dark current electrons generated from the cathode itself will lead to electron back-bombardment of the cathode. Electrons generated elsewhere on the gun body did not strike the cathode area in the simulations performed. In addition, the ion generation and propagation simulation for non-cathode generated dark current electrons showed that ions were confined to the outer portion of the gun cells, except for a small stream of ions near the end of the second cell propagating towards the gun output. None of these ions impacted the cathode. The only apparent negative contribution from the noncathode dark current electrons is the increased vacuum load cause by gas desorption when they strike the gun walls

The accumulated charge on the cathode due to the ions corresponds to $1.6 \times 10^{7}$ ions hitting the cathode per Coulomb of cathode dark current at $1 \times 10^{-10}$ torr, which is slightly more than $50 \%$ of the ion count due to the primary electron beam. Additionally, the ions generated by the dark current impact the cathode at relatively low average kinetic energy. Most of the dark current generated ions impact the cathode early in the simulation (within 10 ns from start) with most of these early impacts occuring at a kinetic energy less than $500 \mathrm{eV}$.

\section{VACUUM TESTS}

To test the feasibility of cooling an RF gun to liquid nitrogen temperatures, we built the test stand shown in Figure 3. It consists of an RF gun, three pumping stations consisting of a $20 \mathrm{~L} / \mathrm{s}$ ion pump and titanium sublimation pump (TSP), two faraday cups, and an RGA for residual gas analysis. A viton seal valve and an orifice were used for initial vacuum tests, after which the orifice was removed. No cathode or plug was present in the cathode port if the gun. This is similar to the test stand used in Reference 2.

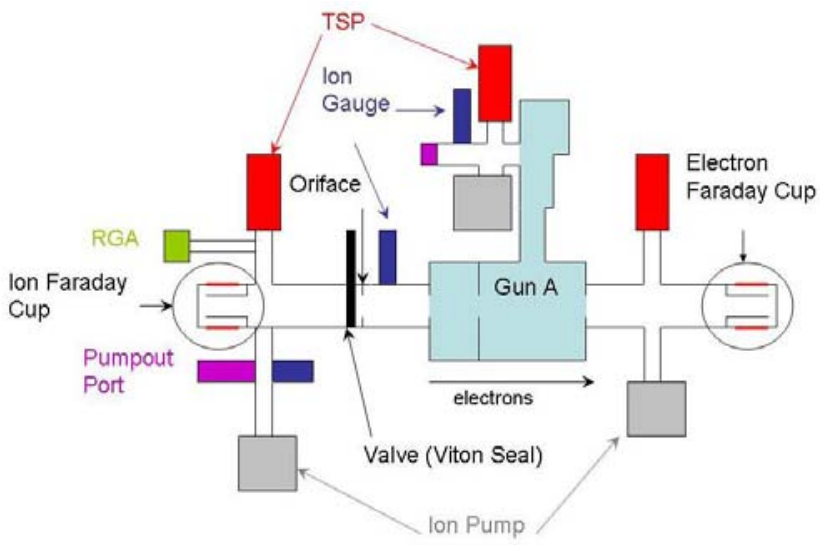

Figure 3: RF Gun test stand.

The out gassing rate of the gun side of the orifice, $Q$, is $8.7 \times 10^{-9}$ torr-L/s, which is typical value for a vacuum system. The pumping speed of each station, S, ranges from 13 to $75 \mathrm{~L} / \mathrm{s}$, decreasing as the TSPs reach their capacity. The orifice was removed after these measurements were taken. The gun was not baked after opening the system because of time constraints.

It is possible to calculate the pressure inside of the gun by knowing the pumping speed of each pumping station, the vacuum conductance from the gun to the station, and the pressure at each station. When the gun was at room temperature, without RF applied, the pressure averaged $3.1 \times 10^{-10}$ torr. The dominant gas was hydrogen, followed by carbon monoxide, carbon dioxide, argon, water, methane, and ethane/ethylene. The argon pressure was anomalously high, likely because the ion pumps were saturated with it. The methane and ethane/ethylene are out gassed when the TSPs are fired. Carbon monoxide and water were an order of magnitude higher than seen in DC guns [5].

We were able to cool the gun to $92 \mathrm{~K}$ by flowing liquid nitrogen through the gun's cooling pipes. Changes in the system pressure as the gun is cooled are due to changes in the pumping speed in the gun from additional cryopumping (assumed to be small), and conductance reductions from a colder gas inside of the gun. These combine to increase the gun pressure at liquid nitrogen temperature to $3.8 \times 10^{-10}$ torr, with a similar residual gas composition. We note that the ion pump was used to measure the pressure near the electron faraday cup, and it was at its lower limit for this case. Pressures on the ion gauges of the system were not, and dropped by a factor of 
2. If the same drop occurred in the electron arm of the system, the pressure in the gun would be unchanged. Installing an ion gauge on this arm is necessary to check this. In addition, measurements of the outgassing rate with the cold gun will allow us to better estimate the gun temperature. Additional measurements of the actual gun pumping speed are necessary to more accurately estimate the pressure inside of the gun.

In order for cryopumping to be truly effective, the temperature of the gun must be low enough so that the vapor pressure of the residual gases is below the desired operating pressure. Temperatures on the order of $20 \mathrm{~K}$ are necessary to reach vapor pressures of $10^{-10}$ torr for most of the gases present in our system.

To measure the out gassing from the RF, RF was applied in 20 to $60 \mu$ s pulses, at a $1 \mathrm{~Hz}$ rep rate. The delivered power was $2 \mathrm{MW}$ for the warm gun and 0.85 MW for the cold gun, which corresponds to an equivalent cathode gradient of $35 \mathrm{MV} / \mathrm{m}$. Less power is required for the cold gun since the conductivity of the copper is higher at lower temperatures.

The pressure rose to $1.1 \times 10^{-9}$ torr with RF applied to the warm gun. This implies that the out gassing rate to the system quadruples because of the RF. The RGA indicated that all of the gases present increased except for hydrogen and water.

The pressure increased to $5.8 \times 10^{-10}$ torr in the cold gun when RF was applied. From this we conclude that the gun outgases a factor of five less when compared to the warm gun. The RGA shows an increase in hydrogen, methane and argon. A slight increase in carbon monoxide is also seen. The other gases show no increase. Carbon dioxide and ethylene are below their melting point at $92 \mathrm{~K}$. It seems the surface heating is not enough to drive the condensed gases from the surface. Argon, methane and ethane are very close to the melting point, and show reduced or no out gassing. We believe that the colder gun condenses some of the gases and the reduced surface heating reduces the out gassing from these gases.

\section{DARK CURRENT}

We measured the electron dark current emerging from the electron port and cathode port of the gun using the faraday cups. The orifice was not in place for these measurements. Figure 4 shows the dark current for the warm and cold gun. The dark current moving in the beam direction is called the forward dark current. The current that would strike the cathode is called the reverse dark current. It was possible to go to higher fields in the cold gun because of the increased cavity $\mathrm{Q}$.

The forward dark current is 5.4 times greater for the cold gun than the warm at $31 \mathrm{MV} / \mathrm{m}$. The reverse dark current is 5.8 times greater in the cold gun than the warm. The reason for the increased dark current in the cold gun is not understood, however is consistent with previous measurements $[2,5]$.

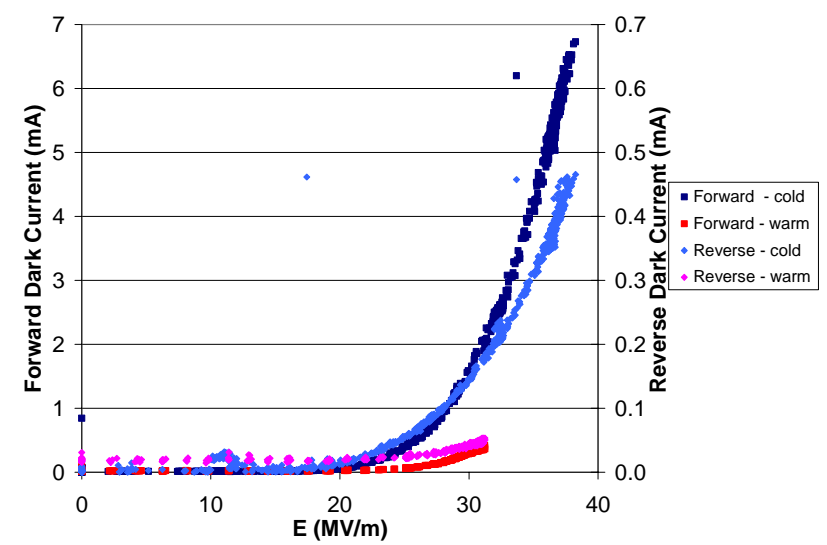

Figure 4: Forward and Reverse Dark currents for the warm and cold guns.

\section{CONCLUSION}

An RF electron gun using an NEA GaAs photocathode can be a promising source for the ILC. However, the question of cathode lifetime in this environment is still an open question. We have produced simulations that show that $\mathrm{H}_{2}$ residual gas pressures of $1 \times 10^{-10}$ torr should be tolerable in an RF gun. In addition, we have measured the out gassing rate of, and the dark current in a cryogenically cooled RF gun.

We plan on investigating ways to further reduce the pressure in the gun. The pumping speed that can be applied to the gun is limited by the vacuum conductance through the gun ports. Reducing the out gassing rate, is the only other way to reduce the pressure. We are investigating techniques such as high temperature baking. Studies are planned to more accurately simulate the environment in the gun based on these measurements.

We have designed a preparation chamber to produce cesiated GaAs cathodes. This will provide us with a way to test these cathodes in an RF gun in the near future.

\section{REFERENCES}

[1] Y.-E. Sun, et. al. "Generation of Angular-Momentum Dominated Electron Beams from a Photoinjector." Phys. Rev. STAB 7 (2004), 123501.

[2] M. Huening. "On Using NEA Cathodes in an RF Gun.” Proceedings of the 2004 European Particle Accelerator Conference, MOPLT11.

[3] B. Goplen et. al., "User-Configurable MAGIC Code for Electromagnetic PIC Calculations,” Comp. Phys. Comm., 87 (1995) 54, and www.mrcwdc.com/Magic/index.html

[4] B.M. Dunham, P Hartmann, R. Kazimi, H. Liu, M.B. Poelker, J.S. Price, P.M. Rutt, W.J. Schneider, and C.K. Sinclair, "Advances in DC Photocathode Electron Guns,” in Advanced Accelerator Concepts, W. Lawson, Ed., AIP Conference Proceedings 472 (1999) 813.

[5] M. Hüning. “Polarized Gun R\&D”. Fermilab Beams Doc \# 1484-v1. 